\title{
Clinical perspectives for the use of total body PET/CT
}

\author{
Ronan Abgral ${ }^{1,2}$. David Bourhis ${ }^{1,2} \cdot$ Pierre-Yves Salaun $^{1,2}$ \\ Published online: 19 March 2021 \\ (C) The Author(s), under exclusive licence to Springer-Verlag GmbH Germany, part of Springer Nature 2021
}

\section{Introduction}

In the last decade, PET scanners have benefited from new technologies such as the TOF system, PSF correction, and SiPM detectors. More recently, the increase of detector number allowed an axial FOV as large as a human body length in a total-body PET (TB-PET) system. Two different scanners called Quadra $(C$ and $\mathrm{uEXPLORER} \subset$ C have been respectively proposed by Siemens Healthineers and United Imaging Healthcare. These TB-PET have two major advantages over actual systems on the market: a gain in detection sensitivity and a possibility of whole-body dynamic multiple organs analysis $[1,2]$. More than reducing drastically the acquisition duration and/or injected activities, these systems open up very interesting clinical perspectives to address specific issues encountered in routine clinical practice and thus improve patient care.

We aimed in this brief communication to highlight some situations for whom a TB-PET might help to settle current clinical issues.

\section{High temporal resolution whole-body dynamic imaging}

4D dynamic PET (dynPET) acquisition methods have been proposed for extracting quantitative parameters from the temporal analysis of the radiotracer distribution in voxels [3, 4]. It allows to estimate kinetic parameters such as the tracer uptake rate $\mathrm{Ki}$ (slope) using a Patlak-based analysis modeling, after a plasma input function estimation from the images $[5,6]$. Several studies have demonstrated its superiority (vs the usual 3D-approach) for

This article is part of the Topical Collection on Technology

Ronan Abgral

ronan.abgral@chu-brest.fr

1 Department of Nuclear Medicine, University Hospital of Brest, 2 avenue Foch, 29609 Brest cedex, France

2 EA GETBO 3878, University of Western Brittany, Brest, France characterizing different solid tumors and distinguishing inflammation vs malignancy $[7,8]$. Nevertheless, the first studies assessing dynPET acquisition only concerned one step (15$20 \mathrm{~cm}$ ), constituting a limit in the evaluation of multiorgans cancerous or inflammatory pathologies.

Recently, multistep dynPET acquisition protocols have been developed, making possible a whole-body (WB) Kibased analysis but with the condition of an iterative bed motion in the PET system and with the limitation of a low temporal sampling (WB in 5-10 min).

New TB-PET prototypes allow to increase the quality of 4Dquantification with excellent temporal sampling (WB in 10-20s), suggesting the resolution of the following clinical research issues.

\section{Differentiation of benign/pathological processes}

\section{Pseudo-progression vs progression to immunotherapy}

Immunotherapy with checkpoint inhibitors (ICI) has been a highly developed therapeutic strategy in recent years in oncology. Particularly, the first studies showed remarkable efficacy of anti-PD1-type molecules (nivolumab, pembrolizumab) in metastatic melanoma $(\mathrm{mM})$ or lung cancer $(\mathrm{mLC})$, with respective response rates of $40 \%[9,10]$ and $20 \%[11,12]$.

Nevertheless, these innovative therapies present certain problems hitherto unknown with chemotherapy and in particular the concept of pseudo-progression (PP). This phenomenon is defined as an initial increase in tumor size or a new lesion appearing, followed by a decrease in tumor burden.

18FDG-PET/CT can be proposed to assess tumor response after treatment by systemic therapy of mM or mLC [13]. However, this particular response profile has led to the establishment of new evaluation criteria in imaging with the integration of the notion of "unconfirmed progression" [14]. Consequently, the real response to treatment must be assessed several weeks after, in order to eliminate with certainty a PP linked to the tumor infiltration by lymphocytes, inducing on the contrary a loss of time in the diagnosis of confirmed progression (CP) with consequences for patient care and public health costs. 
Thus, TB-PET might have an interest in early differentiating the $\mathrm{CP}$ from the PP of such metastatic cancers treated by ICI.

\section{Residual disease vs post-treatment tissue distortions}

For certain cancers, 18FDG-PET/CT shows high diagnostic performance for the post-treatment residual disease (RD) assessment $[15,16]$ because post-radiation or post-surgical tissue changes reduce the specificity of CT or MRI examinations [17]. For example, it can be proposed for the post-treatment evaluation of head-neck or cervical carcinoma [13]. However, a period of 3 months after treatment must be observed for limiting the false positive risk linked to the early local inflammation (LI). Its negative predictive value in these indications is excellent $(\sim 100 \%)$, but the positive predictive value remains imperfect because certain interpretations leave doubt between the minimal RD and the absence of complete LI extinction [18]. Now, as above-mentioned, kinetic parameters extracted from dynPET acquisition are significantly different in malignant and inflammatory processes, thereby an optimized dynamic signal analysis with TB-PET could be of interest to differentiate minimal $\mathrm{RD}$ and post-therapeutic LI of such cancers.

\section{Insufficient suppression of myocardial signal vs inflammatory process}

18FDG-PET/CT can be useful for the diagnosis of inflammatory cardiovascular diseases (CD) such as atherosclerosis, sarcoidosis, or subacute myocarditis [19]. However, it remains relatively low used in clinical routine practice because it has certain limitations.

First of all, despite the recent improvement in double-gated PET and CT acquisition techniques, cardiac motion induces a low tissue-to-blood pool ratio (TBR) in case of moderate inflammatory process with a higher risk of false negative [20]. Moreover, even if the specific cardiac free fatty acid consumption diet has been respected, an insufficient suppression of physiological myocardial uptake (PMU) is found in approximately $1 / 3$ of cases [21]. Recently, the Ki approach in PET has been suggested to be more efficient than the SUV-based one for the differentiation of a real inflammatory process to an insufficient suppression of PMU [20]. In addition to facilitate this physiological/pathological uptake, TB-PET might also play a role for such dedicated cardiovascular PET studies to improve inflammatory CD diagnosis by optimizing TBR quantification.

\section{Simultaneous information collection with tracer cocktail injection}

The concept of "cocktail" injection of PET tracers, consisting in administering 2 radiopharmaceuticals before a single PET acquisition procedure, has already been evaluated [22-24]. However, it has not been implemented in routine practice due to several limitations such as the lack of consensus for dose proportion and the difficulty in separating the signal of both tracers. Recently, a feasibility study has suggested that WB-dynPET could quantitatively extract the two separate tracer kinetics and also analyze different processes [25].

The application of this "two in one" approach in TB-PET might improve the characterization of several cancers in optimizing radiation exposure and patient comfort and in maximizing the cost-effectiveness strategy.

Its high sensitivity could be also a solution to reduce in this single scan the injected dose of "cocktail," as in the following examples.

\section{Bone extension of osteophilic cancers}

PET imaging with various radiopharmaceuticals can provide specific information for osseous remote extension of solid cancers. Indeed, $18 \mathrm{FDG}$ is a marker of glucose metabolism which is often increased in lytic metastasis, whereas $18 \mathrm{FNa}$ is a marker of osteoblastic activity with high potential for detecting condensing metastases. Combining the strengths of the two tracers would also improve osseous metastases detection rates in case of mixed presentation; and separating signal of these two phenomena remains essential for therapeutic and prognostic considerations, FDG-avid lesions being more aggressive [26].

One of the most frequent examples concerns breast cancer. 18FDG-PET/CT is recommended for initial staging in patients with clinical stage $\geq$ IIB [13]. Nevertheless, its performances in osseous extension assessment are lower in case of invasive lobular subtype (around 15\% of breast cancers), due to the mixed presentation of metastatic lesions [26] so that $18 \mathrm{FDG} / 18 \mathrm{FNa}$ cocktail studies would be interesting.

\section{Aggressiveness and secretion of neuroendocrine tumors}

The characterization of neuroendocrine tumors (NETs) can be done by functional PET imaging with different tracers depending on the location of the primary tumor and the histological grade. 68Ga-DOTATOC-PET/CT is thus indicated in G1-G2 NETs while 18FDG PET in G3 NETs [27-29]. However, it has been shown that 18FDG$\mathrm{PET} / \mathrm{CT}$ was very sensitive to detect certain so-called "borderline" G2 tumors with a Ki67index $>10 \%$ [30]. Furthermore, among G1-G2 NETs, some studies have suggested that 18F-DOPA-PET/CT is more effective than somatostatin-receptor imaging for the midgut primary lesions [31]. As a result, patients may have to undergo 2 (even 3) PET scans with different tracers to explore distinct biological phenomena from their disease. 
Aggressiveness and hormone-receptor expression of cancers

In the same way, several new agents targeting hormonereceptors could improve management of hormone-dependent cancers [32]. The most studied was the 18FES for breast cancers presenting estrogen receptors (ER) [33]. This tracer can be used to determine tissue levels of ER expression and thereby map hormone-sensitive lesions.

In this case, a cocktail of $18 \mathrm{FDG} / 18 \mathrm{FES}$ could be important to obtain complementary information for such cancers with high intra-lesion heterogeneity that impact treatment success.

\section{Improvement of intra- and inter-tumoral heteroge- neity assessment}

Many cancers present a high-level inter-tumor and intra-tumor heterogeneity (different cell populations of variable proliferation rates and areas of angiogenesis, hypoxia, necrosis or fibrosis, etc.), making the therapeutic choice difficult [34].

Among them, NETs constitute a network of tumors of common embryological origin but capable of developing in multiple organs and of secreting different hormones, which may thus lead to varied clinical presentations [35]. Moreover, despite its low incidence, more than $25 \%$ of patients are diagnosed at metastatic stage for which numerous treatments with different pharmacokinetics can be proposed (somatostatin analogues, targeted therapies, polychemotherapy, peptidereceptor radionuclide therapy, etc.).

So, it is crucial to search for surrogate markers in PET/CT to early predict treatment response.

The first dynPET studies showed the absence of a linear correlation between SUV and Ki values, suggesting additional quantitative information from kinetic data and opening new perspectives for the prognostic assessment of NETs [36]. But as above-mentioned, these preliminary reports concerned a one-step analysis, a limiting factor for multimetastatic and multiorgans diseases assessment. More recently, digital systems have validated the WB-dynPET approach but with an iterative constraint of bed motion in the machine [37].

The TB-PET could probably improve the predictive value of treatment response in such multimetastatic disease with a WB parametric Ki map reflecting the temporal inter-/intralesions biodistribution of tracer.

\section{Phenotypic or molecular approaches}

\section{Phenotypic imaging of inflammation}

As part of the ICI-induced inflammation during tumor PP, there is a recruitment of $\mathrm{B}$ and $\mathrm{T}$ lymphocytes [38]. On the contrary, in a post-surgical situation, there is an infiltration of inflammatory cells (macrophages and dendritic cells) into the operated site [39]. The post-radiation inflammation is of mixed presentation, the macrophages presenting the antigens of the destroyed tumor cells to the lymphocytes [40].

It is therefore conceivable to hypothesize that $18 \mathrm{FDG}$ uptake is different within these inflammatory processes and that TB-PET, beyond its usefulness for inflammation/tumor differentiation, could become a real phenotypic imaging capable of characterizing different inflammatory phenomena.

Likewise, it would be of potential interest for the differential diagnosis of rheumatic pathologies or systemic diseases mediated by different inflammatory cells.

\section{Molecular imaging of thrombus}

Molecular imaging has emerged as a new method of in vivo imaging allowing better visualization, characterization, and measurement of biological processes.

Venous thromboembolic disease (VTE) represents the 3rd cause of cardiovascular disease. The risk of death from untreated pulmonary embolism (PE) is approximately $30 \%$. Anticoagulant treatment is effective but presents a significant risk of hemorrhage that can also be fatal [41]. For now, pulmonary SPECT lacks surrogate markers of PE recurrence at diagnosis.

The different constituents of the active venous thrombus (i.e., activated platelets, fibrin network) are potential specific targets for molecular imaging that may provide supplementary information for treatment management. In this context, few series have investigated its feasibility in SPECT [42-44] but reported limitations due to the intrinsic performances of system. To date, none of the tracers studied is used in clinical routine, not for lack of specificity but of detection sensitivity.

In this illustrative example, the high-resolution of TB-PET with dynamic analysis capabilities could allow a better characterization of such specific biological processes to improve a disease recurrence prediction and therefore the therapeutic management of patients.

\section{Ultrahigh sensitivity imaging}

\section{Optimization of radiomic analysis}

Tumor heterogeneity characterization is a major issue in oncology to improve therapeutic management and move towards a personalized medicine adapted to each patient. The textural analysis (radiomic) in PET imaging corresponds to an analysis of the spatial distribution of voxels and allows the calculation of numerous indices reflecting this heterogeneity. Nevertheless, the quality of this textural analysis is dependent on the noise (therefore on the intrinsic system performance) and remains limited for the small lesions [45].

The TB-PET could enable image reconstructions with a noise-limited high-resolution matrix, improving especially 
radiomic analysis of small and/or moving lesions such as in the following examples.

\section{Characterization of small size incidentalomas}

Thyroid (TI) and adrenal (AI) incidentalomas are lesions fortuitously discovered on imaging with respective prevalence of around $2 \%$ and $5 \%$. The search for predictive factors of malignancy in PET therefore appears interesting. Numerous studies have sought the interest of several quantitative parameters such as SUV, MTV, and TLG [46, 47], but no real threshold with high diagnostic performance has been proposed. A few studies have examined the radiomic analysis value in TI and AI characterization, concluding its difficult application in routine practice due to their too small size $[47,48]$, that highsensitive TB-PET could solve.

\section{Signal quantification of moving lesions}

Numerous studies have shown the prognostic value of 18FDG-PET textural analysis in solid tumors, including lung cancer [49].

Nevertheless, radiomic analysis is limited for small lesions in the pulmonary bases (partial-volume effect) [50]. Several respiratory gating algorithms have been developed but are not sufficient to overcome these limits [45]. In this context, the TB-PET could allow shorter image acquisitions in apnea (several seconds) to exceed these detrimental effects of respiratory movement on radiomic analysis.

\section{Ultra-fast acquisition for motion correction}

In addition to obvious clinical considerations such as for patients with pain, claustrophobia, or restlessness, possibilities of ultra-fast breath-hold acquisitions in TB-PET might be useful to correct signal processing in moving regions. For example, it could become an interesting quantitative tool to assess more precisely lung function (LF).

Indeed, the physiological tests currently used give a global LF result. And providing spatial information on LF is one of the major challenges in pulmonology. Indeed, having a map of regional LF in ventilation/perfusion (V/Q) could have an impact in different clinical applications.

In the context of PE, several studies have shown that the index of vascular obstruction in V/Q SPECT is a prognostic factor [51]. Moreover, LF mapping could also have an impact on the patient management before surgery or radiotherapy planning of lung cancer [52]. However, SPECT quantification remains relative, and no tool is actually consensual.

The V/Q PET/CT with 68Ga-radiolabeled tracers is a new imaging technique allowing a SUV-based quantification more precise than in SPECT with absolute results [52]. However, respiratory motion remains a major intrinsic limit to a precise, reliable, and reproducible definition of these volumes that TBPET fast acquisitions might solve. As previously mentioned, respiratory synchronization techniques exist in PET but remain limited in case of irregular respiratory rhythm [53].

\section{High precision characterization of cancer micro- environment}

The high sensitivity of TB-PET could provide high-resolution noiseless images, leading to a detectability improvement, especially for low contrast uptakes. Thus, it would enhance images the diagnostic performance of lymph node microinvasion or micro-metastasis of solid cancer, as in the following illustrative situations.

\section{Peritoneal micro-metastasis}

The diagnosis of peritoneal carcinomatosis (PC) is sometimes difficult to establish by imaging, and the lesions may be very small and scattered throughout the abdominopelvic cavity. In the case of ovarian cancer, its evaluation is essential at the initial staging to assess the possibilities of tumor resectability and the interest of neoadjuvant chemotherapy. Currently, two predictive scores are used (Fagotti or Sugarbaker during laparoscopy or laparotomy, respectively) to assess PC but remain probabilistic and imperfect [54], for that TB-PET could provide additional information before treatment decision.

\section{Lymph node micro-invasion}

Conventional CT and/or MRI imaging has limited performance in the assessment of lymph node extension of solid cancers in case of micro-invasion. Functional PET imaging could improve this detection, in particular by using specific tracers of tumor types.

Prostate-specific membrane antigen (PSMA) is a transmembrane glycoprotein specifically overexpressed on the surface of prostate cancer cells. Recent studies report a higher performance of radiolabeled PSMA-ligands PET/CT to conventional imaging for assessing the initial extension of prostate cancers [55]. Nevertheless, it remains imperfect especially for the evaluation of histologically proven lymph node microinfiltration that have an impact on the treatment management.

\section{Imaging of tracer with low abundance emission and/or long half-life}

One of the particularly promising aspects of TB-PET imaging is the possibility of imaging under conditions of very low statistics, by the use of isotopes with low branching ratio and long half-time $(89 \mathrm{Zr}, 64 \mathrm{Cu}, 90 \mathrm{Y})$.

For example, selective internal radiotherapy (SIRT) by intra-arterial injection of $90 \mathrm{Y}$ microspheres is a therapeutic 
alternative for inoperable primary and secondary liver cancers [56]. The most recent studies highlight the importance of the tumor absorbed dose, significantly associated with overall survival, but also of the dose delivered to healthy organs, a source of radiation-induced side effects [57].

It is necessary to precisely anticipate the distribution of 90Y before SIRT by a work-up and to know its distribution after the procedure, in order to predict the treatment efficacy [58]. Currently, imaging before and after SIRT is performed in SPECT. Nevertheless, PET imaging after SIRT is possible, $90 \mathrm{Y}$ being a positron emitter with a very low-emission abundance $(0.0032 \%)$ limiting its use in clinical practice with actual PET systems.

The TB-PET would also allow much more precise absolute quantification through its high sensitivity detection, optimizing the dosimetry approach in such internal radionuclide therapy for even more precise personalized medicine. It could also be an interesting tool to improve SNR in delayed imaging of such long half-time isotopes.

\section{Ultralow-dose imaging for cancer screening}

With the reported significant gain in sensitivity, ultralow-dose imaging procedures could obviously be considered, in particular for pediatric indications.

But in another exiting context, TB-PET could become a real imaging tool for systematic cancer screening (CS) in radically reducing injected activity. Indeed, systematic CS by $\mathrm{CT}$ or PET imaging is currently limited by the irradiation of patient. Recently, a randomized trial showed that lung CS by CT among high-risk persons (current/former smokers) resulted in substantially lower mortality than in no screened one [59]. 18FDG-PET/CT has already been reported as an interesting $\mathrm{CS}$ tool in the management of unprovoked venous thromboembolism [60]. Thus, iterative ultralow-dose TB-PET scans could be considered in the future for a CS strategy in a selective population of patients with risk factors.

\section{Conclusion}

TB-PET technology gives multiple opportunities to address clinical issues currently unsolved in clinical practice and opens up perspectives of use as a low-dose radiation imaging tool for cancer screening.

\section{Declarations}

Ethics approval Institutional Review Board approval was not required because the paper is an editorial.

Conflict of interest The authors declare no competing interests.
Informed consent Not applicable.

\section{References}

1. Surti S, Pantel AR, Karp JS. Total Body PET: Why, how, what for? IEEE Trans Radiat Plasma Med Sci. 2020;4:283-92.

2. Vandenberghe S, Moskal P, Karp JS. State of the art in total body PET. Ejnmmi Phys. 2020;7:35.

3. Karakatsanis NA, Lodge MA, Tahari AK, Zhou Y, Wahl RL, Rahmim A. Dynamic whole-body PET parametric imaging: I. Concept, acquisition protocol optimization and clinical application. Phys Med Biol. 2013;58:7391-418.

4. Karakatsanis NA, Lodge MA, Zhou Y, Wahl RL, Rahmim A. Dynamic whole-body PET parametric imaging: II. Task-oriented statistical estimation. Phys Med Biol. 2013;58:7419-45.

5. Patlak CS, Blasberg RG, Fenstermacher JD. Graphical evaluation of blood-to-brain transfer constants from multiple-time uptake data. J Cereb Blood Flow Metab. 1983;3:1-7.

6. Karakatsanis NA, Zhou Y, Lodge MA, Casey ME, Wahl RL, Zaidi $\mathrm{H}$, et al. Generalized whole-body Patlak parametric imaging for enhanced quantification in clinical PET. Phys Med Biol. 2015;60: 8643-73.

7. Rahmim A, Lodge MA, Karakatsanis NA, Panin VY, Zhou Y, McMillan A, et al. Dynamic whole-body PET imaging: principles, potentials and applications. Eur J Nucl Med Mol Imaging. 2019;46: 501-18.

8. Karakatsanis NA, Casey ME, Lodge MA, Rahmim A, Zaidi H. Whole-body direct 4D parametric PET imaging employing nested generalized Patlak expectation-maximization reconstruction. Phys Med Biol. 2016;61:5456-85.

9. Robert C, Long GV, Brady B, Dutriaux C, Maio M, Mortier L, et al. Nivolumab in previously untreated melanoma without BRAF mutation. N Engl J Med. 2015;372:320-30.

10. Robert C, Schachter J, Long GV, Arance A, Grob JJ, Mortier L, et al. Pembrolizumab versus ipilimumab in advanced melanoma. $\mathrm{N}$ Eng1 J Med. 2015;372:2521-32.

11. Reck M, Rodríguez-Abreu D, Robinson AG, Hui R, Csőszi T, Fülöp A, et al. Pembrolizumab versus chemotherapy for PD-L1positive non-small-cell lung cancer. N Engl J Med. 2016;375: 1823-33.

12. Borghaei H, Paz-Ares L, Horn L, Spigel DR, Steins M, Ready NE, et al. Nivolumab versus docetaxel in advanced nonsquamous nonsmall-cell lung cancer. N Engl J Med. 2015;373:1627-39.

13. Salaün P-Y, Abgral R, Malard O, Querellou-Lefranc S, Quere G, Wartski $\mathrm{M}$, et al. Good clinical practice recommendations for the use of PET/CT in oncology. Eur J Nucl Med Mol Imaging. 2020;47:28-50.

14. Wolchok JD, Hoos A, O’Day S, Weber JS, Hamid O, Lebbé C, et al. Guidelines for the evaluation of immune therapy activity in solid tumors: immune-related response criteria. Clin Cancer Res. 2009;15:7412-20.

15. Viswanathan C, Faria S, Devine C, Patnana M, Sagebiel T, Iyer RB, et al. [18F]-2-Fluoro-2-Deoxy-D-glucose-PET assessment of cervical cancer. Pet Clin. 2018;13:165-77.

16. Helsen N, den Wyngaert TV, Carp L, Stroobants S. FDG-PET/CT for treatment response assessment in head and neck squamous cell carcinoma: a systematic review and meta-analysis of diagnostic performance. Eur J Nucl Med Mol Imaging. 2018;45:1063-71.

17. Lell M, Baum U, Greess H, Nömayr A, Nkenke E, Koester M, et al. Head and neck tumors: imaging recurrent tumor and post-therapeutic changes with CT and MRI. Eur J Radiol. 2000;33:239-47.

18. Gupta T, Master Z, Kannan S, Agarwal JP, Ghsoh-Laskar S, Rangarajan V, et al. Diagnostic performance of post-treatment FDG PET or FDG PET/CT imaging in head and neck cancer: a 
systematic review and meta-analysis. Eur J Nucl Med Mol Imaging. 2011;38:2083.

19. Abgral R, Dweck MR, Trivieri MG, Robson PM, Karakatsanis N, Mani V, et al. Clinical utility of combined FDG-PET/MR to assess myocardial disease. JACC Cardiovasc Imaging. 2017;10:594-7.

20. Dweck MR, Abgral R, Trivieri MG, Robson PM, Karakatsanis N, Mani V, et al. Hybrid magnetic resonance imaging and positron emission tomography with fluorodeoxyglucose to diagnose active cardiac Sarcoidosis. JACC Cardiovasc Imaging. 2018;11:94-107.

21. Joshi NV, Vesey AT, Williams MC, Shah ASV, Calvert PA, Craighead FHM, et al. 18F-fluoride positron emission tomography for identification of ruptured and high-risk coronary atherosclerotic plaques: a prospective clinical trial. Lancet. 2014;383:705-13.

22. Iagaru A, Mittra E, Yaghoubi SS, Dick DW, Quon A, Goris ML, et al. Novel strategy for a cocktail 18F-fluoride and 18F-FDG PET/ $\mathrm{CT}$ scan for evaluation of malignancy: results of the pilot-phase study. J Nucl Med. 2009;50:501-5.

23. Simoncic U, Perlman S, Liu G, Jeraj R. Optimizing an $18 \mathrm{~F}-\mathrm{NaF}$ and 18F-FDG cocktail for PET assessment of metastatic castrationresistant prostate cancer. Nucl Med Commun. 2015;36:1174-80.

24. Roop MJ, Singh B, Singh H, Watts A, Kohli PS, Mittal BR, et al. Incremental value of cocktail $18 \mathrm{~F}-\mathrm{FDG}$ and $18 \mathrm{~F}-\mathrm{NaF}$ PET/CT over 18F-FDG PET/CT alone for characterization of skeletal metastases in breast cancer. Clin Nucl Med. 2017;42:335-40.

25. Karakatsanis NA, Abgral R, Trivieri MG, Dweck MR, Robson PM, Calcagno C, et al. Hybrid PET- and MR-driven attenuation correction for enhanced $18 \mathrm{~F}-\mathrm{NaF}$ and $18 \mathrm{~F}-\mathrm{FDG}$ quantification in cardiovascular PET/MR imaging. J Nucl Cardiol. 2020;27:1126-41.

26. Groheux D, Giacchetti S, Moretti J-L, Porcher R, Espié M, Lehmann-Che J, et al. Correlation of high 18F-FDG uptake to clinical, pathological and biological prognostic factors in breast cancer. Eur J Nucl Med Mol Imaging. 2011;38:426-35.

27. Barrio M, Czernin J, Fanti S, Ambrosini V, Binse I, Du L, et al. The impact of somatostatin receptor-directed PET/CT on the management of patients with neuroendocrine tumor: a systematic review and meta-analysis. J Nucl Med. 2017;58:756-61.

28. Graham MM, Gu X, Ginader T, Breheny P, Sunderland JJ. 68GaDOTATOC imaging of neuroendocrine tumors: a systematic review and meta-analysis. J Nucl Med. 2017;58:1452-8.

29. Partelli S, Rinzivillo M, Maurizi A, Panzuto F, Salgarello M, Polenta V, et al. The role of combined 68Ga-DOTANOC and $18 \mathrm{FDG} \mathrm{PET} / \mathrm{CT}$ in the management of patients with pancreatic neuroendocrine tumors. Neuroendocrinology. 2015;100:293-9.

30. Abgral R, Leboulleux S, Déandreis D, Aupérin A, Lumbroso J, Dromain C, et al. Performance of 18Fluorodeoxyglucose-positron emission tomography and somatostatin receptor scintigraphy for high Ki67 ( $\geq 10 \%)$ well-differentiated endocrine carcinoma staging. J Clin Endocrinol Metab. 2011;96:665-71.

31. Ansquer C, Touchefeu Y, Faivre-Chauvet A, Leux C, Bras ML, Régenet N, et al. Head-to-head comparison of 18F-DOPA PET/CT and 68Ga-DOTANOC PET/CT in patients with midgut neuroendocrine tumors. Clin Nucl Med. 2020.

32. Katzenellenbogen JA. PET imaging agents (FES, FFNP, and FDHT) for estrogen, androgen, and progesterone receptors to improve management of breast and prostate cancers by functional imaging. Cancers. 2020;12:2020.

33. Evangelista L, Dieci MV, Guarneri V, Conte PF. 18FFluoroestradiol positron emission tomography in breast cancer patients: systematic review of the literature \& meta-analysis. Curr Radiopharm. 2016;9:244-57.

34. Dagogo-Jack I, Shaw AT. Tumour heterogeneity and resistance to cancer therapies. Nat Rev Clin Oncol. 2018;15:81-94.

35. Modlin IM, Oberg K, Chung DC, Jensen RT, de Herder WW, Thakker RV, et al. Gastroenteropancreatic neuroendocrine tumours. Lancet Oncol. 2008;9:61-72.
36. Velikyan I, Sundin A, Sörensen J, Lubberink M, Sandström M, Garske-Román U, et al. Quantitative and qualitative intrapatient comparison of 68Ga-DOTATOC and 68Ga-DOTATATE: net uptake rate for accurate quantification. J Nucl Med. 2014;55:204-10.

37. Thuillier P, Bourhis D, Karakatsanis N, Schick U, Metges JP, Salaun P-Y, et al. Diagnostic performance of a whole-body dynamic 68GA-DOTATOC PET/CT acquisition to differentiate physiological uptake of pancreatic uncinate process from pancreatic neuroendocrine tumor. Medicine. 2020;99:e20021.

38. Ma Y, Wang Q, Dong Q, Zhan L, Zhang J. How to differentiate pseudoprogression from true progression in cancer patients treated with immunotherapy. Am J Cancer Res. 2019;9:1546-53.

39. Arias J-I, Aller M-A, Arias J. Surgical inflammation: a pathophysiological rainbow. J Transl Med. 2009;7:19.

40. Schaue D, Micewicz ED, Ratikan JA, Xie MW, Cheng G, McBride WH. Radiation and inflammation. Semin Radiat Oncol. 2015;25:4 10 .

41. Konstantinides SV, Meyer G, Becattini C, Bueno H, Geersing G-J, Harjola V-P, et al. 2019 ESC guidelines for the diagnosis and management of acute pulmonary embolism developed in collaboration with the European Respiratory Society (ERS)the task force for the diagnosis and management of acute pulmonary embolism of the European Society of Cardiology (ESC). Eur Heart J. 2019;41:543-603.

42. Douketis JD, Ginsberg JS, Haley S, Julian J, Dwyer M, Levine M, et al. Accuracy and safety of $99 \mathrm{mTc}$-labeled anti-D-dimer (DI80B3) fab' fragments (ThromboView $\left.{ }^{\circledR}\right)$ in the diagnosis of deep vein thrombosis: a phase II study. Thromb Res. 2012;130:381-9.

43. Taillefer R, Edell S, Innes G, Lister-James J. Acute thromboscintigraphy with $(99 \mathrm{~m}) \mathrm{Tc}$-apcitide: results of the phase 3 multicenter clinical trial comparing $99 \mathrm{mTc}$-apcitide scintigraphy with contrast venography for imaging acute DVT. Multicenter trial investigators. J Nucl Med. 2000;41:1214-23.

44. Brighton T, Janssen J, Butler SP. Aging of acute deep vein thrombosis measured by radiolabeled $99 \mathrm{mTc}-\mathrm{rt}-\mathrm{PA}$. J Nucl Med. 2007;48:873-8.

45. Reuzé S, Schernberg A, Orlhac F, Sun R, Chargari C, Dercle L, et al. Radiomics in nuclear medicine applied to radiation therapy: methods, pitfalls, and challenges. Int J Radiat Oncol Biol Phys. 2018;102:1117-42.

46. Thuillier P, Roudaut N, Crouzeix G, Cavarec M, Robin P, Abgral $R$, et al. Malignancy rate of focal thyroid incidentaloma detected by FDG PET-CT: results of a prospective cohort study. Endocr Connect. 2017;6:413-21.

47. Thuillier P, Bourhis D, Schick U, Alavi Z, Guezennec C, Robin P, et al. Diagnostic value of PET textural indices for malignancy of FDG-avid adrenal lesions. Q J Nucl Medicine Mol Imaging. 2019.

48. Thuillier P, Bourhis D, Roudaut N, Crouzeix G, Alavi Z, Schick U, et al. Diagnostic value of FDG PET-CT quantitative parameters and Deauville-like 5 point-scale in predicting malignancy of focal thyroid incidentaloma. Front Med. 2019;6:24.

49. Dissaux G, Visvikis D, Da-ano R, Pradier O, Chajon E, Barillot I, et al. Pretreatment 18 F-FDG PET/CT radiomics predict local recurrence in patients treated with stereotactic body radiotherapy for early-stage non-small cell lung cancer: a multicentric study. J Nucl Med. 2019;61:814-20.

50. Grootjans W, Tixier F, van der Vos CS, Vriens D, Rest CCL, Bussink J, et al. The impact of optimal respiratory gating and image noise on evaluation of Intratumor heterogeneity on 18F-FDG PET imaging of lung cancer. J Nucl Med. 2016;57:1692-8.

51. Tromeur C, Sanchez O, Presles E, Pernod G, Bertoletti L, Jego P, et al. Risk factors for recurrent venous thromboembolism after unprovoked pulmonary embolism: the PADIS-PE randomised trial. Eur Respir J. 2018;51:1701202.

52. Roux P-YL, Hicks RJ, Siva S, Hofman MS. PET/CT lung ventilation and perfusion scanning using Galligas and Gallium-68-MAA. Semin Nucl Med. 2018;49:71-81. 
53. Roux P-YL, Robin P, Salaun P-Y. New developments and future challenges of nuclear medicine and molecular imaging for pulmonary embolism. Thromb Res. 2018;163:236-41.

54. Varnoux C, Huchon C, Bats AS, Bensaid C, Achouri A, Nos C, et al. Diagnostic accuracy of hand-assisted laparoscopy in predicting resectability of peritoneal carcinomatosis from gynecological malignancies. Eur J Surg Oncol. 2013;39:774-9.

55. Perera M, Papa N, Roberts M, Williams M, Udovicich C, Vela I, et al. Gallium-68 prostate-specific membrane antigen positron emission tomography in advanced prostate cancer - updated diagnostic utility, sensitivity, specificity, and distribution of prostatespecific membrane antigen-avid lesions: a systematic review and meta-analysis. Eur Urol. 2019;77:403-17.

56. Edeline J, Gilabert M, Garin E, Boucher E, Raoul J-L. Yttrium-90 microsphere radioembolization for hepatocellular carcinoma. Liver Cancer. 2015;4:16-25.

57. Garin E, Tselikas L, Guiu B, Chalaye J, Edeline J, de Baere T, et al. Personalised versus standard dosimetry approach of selective internal radiation therapy in patients with locally advanced hepatocellular carcinoma (DOSISPHERE-01): a randomised, multicentre, open-label phase 2 trial. Lancet Gastroenterol Hepatol. 2021;6: $17-29$.

58. Salem R, Padia SA, Lam M, Bell J, Chiesa C, Fowers K, et al. Clinical and dosimetric considerations for Y90: recommendations from an international multidisciplinary working group. Eur J Nucl Med Mol Imaging. 2019;46:1695-704.

59. de Koning HJ, van der Aalst CM, de Jong PA, Scholten ET, Nackaerts K, Heuvelmans MA, et al. Reduced lung-cancer mortality with volume CT screening in a randomized trial. N Engl J Med. 2020;382:503-13.

60. Robin P, Roux P-YL, Planquette B, Accassat S, Roy P-M, Couturaud $\mathrm{F}$, et al. Limited screening with versus without $18 \mathrm{~F}$ fluorodeoxyglucose PET/CT for occult malignancy in unprovoked venous thromboembolism: an open-label randomised controlled trial. Lancet Oncol. 2016;17:193-9.

Publisher's note Springer Nature remains neutral with regard to jurisdictional claims in published maps and institutional affiliations. 